\title{
Evaluación del Programa para la Detección, Diagnóstico y Tratamiento Integral de la Depresión en atención primaria, en Chile
}

\author{
Rubén Alvarado, ${ }^{1}$ Jorge Vega, ${ }^{2}$ Gabriel Sanhueza ${ }^{3}$ y María Graciela Muñoz ${ }^{4}$
}

Forma de citar

Alvarado R, Vega J, Sanhueza G, Muñoz MG. Evaluación del Programa para la Detección, Diagnóstico y Tratamiento Integral de la Depresión en atención primaria, en Chile. Rev Panam Salud Publica. 2005; 18(4/5):278-86.

RESUMEN Objetivo. Realizar una evaluación preliminar del Programa para la Detección, Diagnóstico y Tratamiento Integral de la Depresión, instituido por el Ministerio de Salud de Chile.

Métodos. Se realizó un seguimiento de tres meses de un grupo de mujeres que ingresaron en este programa en ocho centros de atención primaria de tres regiones de Chile. La muestra inicial estuvo constituida por 229 personas, de las cuales 169 pudieron entrevistarse a los tres meses. Se estudiaron variables sociodemográficas, factores de riesgo psicosocial, síntomas ansiosos y somatomorfos y el grado de satisfacción con la atención recibida. El cuadro depresivo se evaluó con el Inventario de Depresión de Beck. Se analizaron los tratamientos e intervenciones prescritos a las mujeres.

Resultados. La decisión de ingresar a las pacientes en el programa resultó pertinente, ya que 95,2\% tenían un cuadro depresivo. La concordancia en cuanto a la gravedad del cuadro clínico fue cercana a $50 \%$, con una tendencia a subestimarla.

La tasa de abandono del programa fue de 19,5\% a los tres meses de seguimiento. Las mujeres que se retiran tienden a presentar un cuadro clínico menos complejo (sintomas ansiosos y somatomorfos menos intensos), así como un menor número de factores de riesgo psicosocial y una menor satisfacción con la atención recibida.

Entre las pacientes del programa se observó buena adherencia al tratamiento farmacológico $(73,3 \%)$ y regular a la psicoterapia individual $(47,4 \%)$ y a la intervención grupal $(37,8 \%)$. Se observó un descenso significativo en la intensidad de los síntomas depresivos a los tres meses, el cual fue mayor entre las que presentaban cuadros más graves. Se encontró también un descenso significativo de los sintomas ansiosos y somatomorfos.

Conclusión. La evaluación preliminar indica que el programa está cumpliendo con los objetivos propuestos, si bien parece necesario desarrollar estrategias específicas para mejorar la precisión en el diagnóstico clínico y el cumplimiento de los tratamientos ofrecidos.

Palabras clave Evaluación de programas; trastorno depresivo.

Escuela de Salud Pública, Facultad de Medicina, Universidad de Chile. Toda correspondencia deberá dirigirse a: Rubén Alvarado, Escuela de Salud Pública, Facultad de Medicina, Universidad de Chile. Independencia 938, Santiago, Chile. Teléfono: (56-2) 678 6133; Correo electrónico: ralvarado@ machi.med.uchile.cl
2 Unidad de Salud Mental, Ministerio de Salud, Santiago, Chile.

3 Escuela de Salud Pública, Facultad de Medicina, Universidad de Chile, Santiago, Chile.

4 Departamento de Atención Primaria, Ministerio de Salud, Santiago, Chile.
Se han logrado importantes adelantos en el tratamiento de la depresión, como lo demuestra la amplia variedad de fármacos antidepresivos e intervenciones psicoterapéuticas 
y educativas útiles con que hoy se cuenta.

Se tienen pruebas firmes de la eficacia de los medicamentos antidepresivos (1). En diversas revisiones sistemáticas se ha demostrado la superioridad de varios tipos de estos agentes, en comparación con el placebo, para reducir los síntomas del trastorno depresivo de diversas intensidades. Es por ello que en varias guías de práctica clínica se recomienda su empleo (2). Las diferentes familias de fármacos antidepresivos muestran eficacia clínica similar, si bien varían el perfil y la intensidad de sus efectos secundarios (1).

Por otra parte, existen también pruebas importantes de que la terapia cognitiva es mejor que el placebo para reducir los síntomas en cuadros depresivos de grados leve y moderado (1), lo que determina que se recomiende su aplicación (2, 3). Aunque con menor certeza, se ha afirmado lo mismo de la terapia interpersonal (1). En otro orden, se ha observado que los tratamientos psicológicos y los medicamentos antidepresivos ofrecen eficacia semejante para el tratamiento de cuadros depresivos leves y moderados, sin que ofrezca ventaja la combinación de ambos tipos de tratamiento (1). No obstante, hay pruebas de que esa combinación ofrece mayor eficacia en cuadros graves (1).

Sin embargo, la eficacia demostrada de esas intervenciones en el tratamiento de los cuadros depresivos no siempre se refleja en la práctica clínica cotidiana. Por una parte, hay diversos factores que afectan a la validez externa de los resultados obtenidos en los ensayos clínicos controlados y aleatorizados $(4,5)$. Estos factores pueden agruparse en tres tipos: a) los que dependen del médico (capacidad para identificar los casos, conocimientos sobre el tratamiento, habilidad para detectar creencias particulares en sus pacientes y manejar adecuadamente la relación con ellos); b) los que dependen del paciente (creencias específicas en torno a la enfermedad y los tratamientos, que determinan el cumplimiento de las instrucciones médicas, la valoración de los servicios recibidos y el grado de satisfacción obtenido), y c) los referentes a la forma en que se organiza la atención (disponibilidad de fármacos, capacitación de los profesionales, materiales psicoeducativos de apoyo, sistemas de seguimiento de casos). Todos estos factores determinan la efectividad que se logra en la atención clínica que se ofrece en el ámbito comunitario.

Lo anterior ha motivado la formulación de programas de diversos tipos que buscan mejorar la eficacia de los tratamientos mediante el fortalecimiento de las capacidades del médico, el fomento de la adhesión del paciente a su tratamiento o cambios en la forma en que se brinda la atención (6-10). Dos revisiones sistemáticas recientes han mostrado que los programas de este tipo mejoran mucho el cuadro clínico (los síntomas depresivos), la calidad de las prescripciones médicas (adherencia a los protocolos de atención clínica), el cumplimiento de las instrucciones prescritas y la satisfacción del paciente con el servicio prestado $(11,12)$.

En Chile, Araya et al. (13) han evaluado la efectividad de una intervención multicomponente en la atención primaria (uso de fármacos antidepresivos, intervención grupal psicoeducativa y seguimiento estructurado $\mathrm{y}$ sistemático), en contraste con los métodos tradicionales. Se trata de un ensayo clínico controlado y aleatorizado que se desarrolla en el ámbito comunitario. A los tres meses de seguimiento estos investigadores observaron entre ambos grupos una diferencia no significativa en la reducción de los síntomas depresivos (según la Hamilton Depression Rating Scale), la cual, no obstante, resultó estadísticamente significativa a los seis meses, en favor del grupo con la intervención de varios componentes. En forma secundaria se evaluaron cuatro aspectos del deterioro funcional, utilizando la versión abreviada de 36 preguntas del cuestionario de salud de Medical Outcomes Trust (Health Survey $S F-36)$. En comparación con el grupo de referencia, el grupo de intervención mostró mejores resultados a los tres y a los seis meses. Las conclusiones de este estudio motivaron la puesta en marcha de un programa nacional que incorporó entre sus actividades la interven- ción grupal psicoeducativa ahí propuesta; trabajo grupal para seis a ocho personas en seis sesiones, conforme a un programa estructurado en torno a contenidos educativos sobre la depresión y su tratamiento.

\section{El Programa para la Detección, Diagnóstico y Tratamiento Integral de la Depresión (PDTID) en la atención primaria, en Chile}

El Ministerio de Salud de Chile ha desarrollado un programa de alcance nacional (PDTID) encaminado a optimizar la detección, el diagnóstico y el tratamiento de los trastornos depresivos en el nivel primario de atención. Actualmente, el programa se extiende a todos los centros de atención primaria de la salud (APS) del país (14).

En cuanto a su organización, el PDTID funciona de la siguiente manera. Cualquier profesional del centro puede realizar la detección de los casos probables durante sus actividades habituales de atención. Todo caso probable se deriva a un médico o psicólogo del propio centro, que se encarga entonces de hacer el diagnóstico y, en su caso, de efectuar el ingreso en un programa estructurado de atención. Se realiza un diagnóstico clínico conforme a los criterios de la Clasificación Internacional de Enfermedades, décima revisión (CIE-10), se verifica la gravedad del trastorno y se estudia un conjunto de condiciones que se consideran de riesgo psicosocial (según el consenso de expertos nacionales). Si el cuadro clínico apunta al diagnóstico de depresión grave, se remite a la persona a un centro en el que brinda atención especializada un equipo muldisciplinario dirigido por un psiquiatra. De otro modo, si el diagnóstico corresponde a depresión leve o moderada, se ingresa al paciente en un programa de atenciones que sigue los criterios de una guía de práctica clínica (14).

La atención que se ofrece como parte del PDTID en los centros de APS establece consultas de seguimiento cada dos semanas y evaluaciones por profesionales de diversas áreas (médicos, psicólogos, enfermeras y trabajadores 
sociales) con capacitación apropiada. De acuerdo con la gravedad del problema clínico, se ofrecen distintas opciones que pueden prescribirse por separado o en combinación: fármacos antidepresivos, psicoterapia individual e intervención grupal. Si los resultados clínicos son insuficientes se valora la conveniencia de una evaluación conjunta en el centro de APS del equipo local, con la participación de un psiquiatra consultor. Si el problema persiste, se deriva al paciente a un centro especializado para continuar su atención (14). Cuando se logra una buena respuesta, el paciente se mantiene en seguimiento en su centro de APS por un período mínimo de seis meses (14).

El presente estudio se enmarca dentro de un proyecto más amplio destinado a realizar una evaluación preliminar del PDTID en su etapa de puesta en marcha, de agosto de 2001 a marzo de 2002, con la finalidad de generar información que permita mejorar su funcionamiento y su efectividad. La evaluación fue realizada por un equipo académico de la Escuela de Salud Pública de la Universidad de Chile, que es externo a la gestión nacional o local del PDTID. En esta etapa de la evaluación del PDTID, la estrategia general abarcó tres componentes: a) el seguimiento de una cohorte de pacientes que ingresaron a tratamiento durante los primeros tres meses, b) un estudio del funcionamiento de los equipos de cada centro de atención primaria de la salud (APS), de la forma en que aplican el protocolo de atención y de su coordinación con niveles de atención de mayor complejidad, y c) un análisis económico del funcionamiento del PDTID en cada centro de atención primaria.

El presente trabajo reúne la información obtenida durante el seguimiento de la cohorte de pacientes, con especial atención a tres aspectos específicos:

- La pertinencia del ingreso de las pacientes y sus características clínicas,

- La tasa de abandono en las primeras semanas y los factores asociados, y

- El seguimiento del tratamiento y del cambio en el cuadro clínico a los tres meses.

\section{MATERIAL Y MÉTODO}

En la evaluación del PDTID se estableció un diseño de tipo pre-post (ingreso y tres meses de seguimiento) para el estudio de una muestra de mujeres con diagnóstico de trastorno depresivo que ingresaron en el programa en sus respectivos centros de APS.

\section{Universo y muestra}

El universo se compuso de las mujeres que ingresaron en el PDTID en los centros de APS urbanos donde habitualmente reciben sus servicios de salud. La muestra se extrajo de ocho centros de APS de cuatro ciudades del país (cuatro centros en Santiago, dos en Valparaíso, uno en Concepción y uno en Talcahuano). La selección de centros de diferentes regiones permitió abarcar la diversidad nacional (en términos de población, geografía y asistencia sanitaria), lo que garantiza una buena representatividad de la muestra.

Las mujeres se incorporaban al estudio al momento en que ingresaban en el programa de tratamiento, tras obtenerse su consentimiento fundamentado. Se admitieron todas las que ingresaron en el PDTID en los centros seleccionados durante los meses de agosto y septiembre de 2001; no se hicieron exclusiones. Se entrevistaba a las pacientes al ingreso y al cabo de tres meses, ya sea que continuaran en seguimiento o se hubieran retirado.

Se decidió incluir en el estudio solamente a mujeres, lo que significa un control previo de la variable "sexo" mediante restricción durante el ingreso de posibles casos. La decisión se basó en que la gran mayoría de los pacientes que ingresan en este programa son de sexo femenino (más de $90 \%$ ) y en la intención formal de evitar un análisis de género que, aun siendo de gran importancia, añadiría complejidad excesiva al diseño de investigación.

Por otra parte, se establecieron tres criterios de exclusión: presencia de un déficit sensorial que imposibilitara la comunicación, de un déficit cognitivo moderado a grave y la existencia concomitante de embarazo (para evitar la inclusión de cuadros depresivos relacionados con la gestación).

Originalmente se calculó un tamaño de muestra de $246(P=0,2)$ para obtener un nivel de confianza de $95 \%$ y un error de 5\%. Finalmente, se obtuvo una muestra de 229 casos.

\section{Características de la muestra}

El promedio de edad fue de 43,6 años, con límites de 20 y 73 años. Una proporción de $63,7 \%$ de las mujeres tenía pareja estable $(50,2 \%$ estaban casadas y $13,5 \%$ en relación de convivencia), 14,0\% eran solteras, $14,4 \%$ separadas y $7,9 \%$ viudas. Una proporción de 19,2\% no tenía hijos y 23,1\% tenía tres o más. La mediana del número de hijos fue de 2 .

Casi todas las participantes residían en zonas urbanas $(98,7 \%)$. Al momento de la entrevista inicial, $71,2 \%$ no trabajaban fuera de casa. De las restantes, solo $51,1 \%$ tenían empleo estable. Respecto a escolaridad, 48,7\% completaron la enseñanza básica y 37,3\% la secundaria; la mediana fue de 8 años de estudios.

En $169(73,8 \%)$ de las 229 mujeres que conformaron la muestra inicial logró obtenerse la segunda entrevista de seguimiento. La diferencia se explica por un grupo que no acudió a la segunda cita ni pudo localizarse en su domicilio. En las pruebas de hipótesis que se realizaron para cotejar las características clínicas y psicosociales de ambos grupos no se encontraron diferencias significativas en edad, estado civil, escolaridad o situación laboral ni tampoco en ninguno de los factores psicosociales de riesgo evaluados ni en el cuadro clínico (puntuación en el Inventario de Depresión de Beck, IDB).

\section{Instrumentos utilizados}

De las entrevistas de evaluación se encargaron mujeres profesionales, externas a los centros evaluados, que recibieron capacitación especial para esta tarea. Se utilizaron los instrumentos que se describen a continuación:

a) Encuesta de datos personales (precodificada y con validación semántica previa). 
b) Lista de factores de riesgo psicosocial (14), consistente en 15 eventos o condiciones personales, familiares o sociales, considerados de riesgo psicosocial según consenso de expertos para el país y que deberían evaluarse sistemáticamente al momento de ingresar un paciente al PDTID.

c) El Inventario de Depresión de Beck (IDB), instrumento de 21 preguntas con respuestas precodificadas (15), de uso extenso en todo el mundo para evaluar la intensidad de los cuadros depresivos y cuyas propiedades psicométricas se han estudiado a fondo $(16,17)$. Puede utilizarse en forma dimensional (como variable continua), o bien aplicando las categorías que sugieren sus autores (desde ausencia de depresión hasta depresión grave) (17). A pesar de las limitaciones que caracterizan a los instrumentos dimensionales cuando se utilizan en forma categórica, se decidió usar el IDB porque permite asumir ambas perspectivas de análisis.

d) Una escala para medir síntomas ansiosos y somatoformes (18), compuesta de 24 preguntas precodificadas que permiten diferenciar los dos tipos de síntomas (subescala de trastornos de ansiedad y subescala de trastornos somatoformes) y que puede utilizarse en forma dimensional o categórica; en este último caso se distinguen tres categorías: sin cuadro clínico, con cuadro subclínico y con trastorno clínico. Se elaboró, validó y estandarizó en el país, tomando como referencia los criterios diagnósticos de la tercera edición revisada del Manual de Diagnóstico y Estadística de los Trastornos Mentales (DSM III-R) pertinentes a estos grupos de trastornos.

e) El grado de satisfacción con la atención recibida, que se evaluó con tres escalas de Likert (valores de 1 a 10, desde muy malo hasta muy bueno) en términos del tratamiento indicado, los lugares donde se recibió la atención y el trato personal que brindaron los profesionales a cargo.

f) Una encuesta para evaluar la adherencia a las intervenciones y las causas de incumplimiento.

\section{Análisis de los datos}

El análisis estadístico se realizó con el programa EPI-INFO, versión 6.04. Para evaluar la pertinencia y la precisión del diagnóstico formulado se evaluó la concordancia entre este y la puntuación obtenida en el IDB, utilizando una perspectiva categórica con este último instrumento (17). En este caso el análisis fue de tipo descriptivo.

Para estudiar los factores relacionados con el abandono del programa (variable dicotómica), las variables categóricas se sometieron a pruebas de ji cuadrada y las variables continuas a la prueba de la $t$ de Student para muestras independientes. Cuando no hubo homogeneidad en las varianzas (prueba de Bartlett) se utilizó el resultado obtenido en la prueba de Kruskal-Wallis.

Para evaluar la adherencia a las intervenciones se hizo primeramente una descripción de la frecuencia con que se prescribió cada una. Luego, para establecer si esta prescripción obedecía a criterios relacionados con la intensidad del cuadro depresivo, los promedios en el IDB se compararon al ingreso por medio de una prueba de la $t$ de Student o un análisis de varianza (según el caso). Por último, se estudió la frecuencia con que las pacientes siguen adecuadamente la prescripción de antidepresivos o de las intervenciones psicosociales.

Para analizar las variaciones en las puntuaciones pre y post en el IDB se utilizó la prueba de la $t$ de Student para muestras dependientes, donde la hipótesis alternativa fue un descenso mayor de 0 en la puntuación. En todos los casos se aceptó un valor $P<0,05$ para rechazar la hipótesis de nulidad.

\section{RESULTADOS}

\section{Pertinencia del ingreso de las pacientes y sus características clínicas}

El primer aspecto que interesaba constatar era si las pacientes que se incorporaban al programa efectivamente cumplían los criterios para el diagnóstico de trastorno depresivo. Se encon- tró que solo 4,8\% del total de $229 \mathrm{mu}$ jeres presentaban una puntuación baja en el IDB (menos de 10 puntos), lo que corresponde a ausencia de depresión (18). Este dato confirmó que la gran mayoría de las pacientes incorporadas al programa por los equipos de los centros de APS padecían un trastorno depresivo.

En cuanto a la concordancia en el diagnóstico, la puntuación en el IDB se comparó con los registros de las fichas clínicas. En 141 de los 229 casos $(61,6 \%)$ se había registrado la gravedad del trastorno depresivo diagnosticado. En 20 de estas mujeres se formuló el diagnóstico de depresión leve y en 121 el de depresión moderada (14,2\% y 85,8\%, respectivamente). No se encontró ningún registro con el diagnóstico de depresión grave.

En el cuadro 1 se muestra la comparación entre los resultados en el IDB y el diagnóstico que se registró en la ficha clínica. En 40,0\% del grupo con diagnóstico de depresión leve se encontró una puntuación equivalente a ese rango, en $45,0 \%$ hubo subestimación de la gravedad del cuadro y en 15,0\% una sobrestimación. En cuanto al grupo con diagnóstico de depresión moderada, en 51,2\% fue concordante con las categorías correspondientes en el IDB, en $45,5 \%$ se subestimó la gravedad y en 3,3\% se sobrestimó.

Los corolarios de este apartado son: a) la gran mayoría de las pacientes que ingresan en este programa presentan un cuadro depresivo, b) la concordancia respecto a la gravedad del cuadro clínico es cercana a 50\% (más alta cuando la gravedad es mayor), y c) se aprecia una tendencia a subestimar la gravedad del trastorno en el diagnóstico que se anota en la ficha clínica.

\section{Tasa de abandono y factores relacionados}

De las 169 mujeres que conformaron el grupo de seguimiento, $33(19,5 \%)$ habían abandonado el programa de tratamiento en el momento de la segunda evaluación (a los tres meses). Ninguna de ellas recibía tratamiento en otro lugar. 
De estas pacientes, $42,4 \%$ atribuyeron el abandono a razones personales, como escasez de tiempo u otras cosas que hacer. Una proporción de 30,3\% relacionó su abandono con las características del tratamiento que se le ofrecía, especialmente la participación en grupos de apoyo o el uso de fármacos para tratar este tipo de problemas. No indicó los motivos de la interrupción una proporción de $18,2 \%$.

El cuadro 2 muestra la comparación entre el subgrupo que abandonó el programa y el que continuó asistiendo.

Respecto a la edad, las pacientes que abandonan el programa son significativamente más jóvenes que las que continúan en él. No se encuentran diferencias estadísticas en lo referente a estado civil, escolaridad ni estado ocupacional.

Las mujeres que abandonan el programa presentan un menor número de factores psicosociales de riesgo. Además, al comparar la frecuencia de cada uno de estos en particular, se apreció una diferencia estadísticamente significativa en el porcentaje de conflicto familiar grave y violencia intrafamiliar; lo refirieron $26,4 \%$ de las que se mantenían en el programa, en contraste con 5,7\% de las que lo abandonaron $(P=0,0185)$. Asimismo, las pacientes que permanecían en el programa mostraron una mayor tendencia al consumo de alcohol o drogas y a tener conflictos laborales, si bien las diferencias no alcanzaron significación estadística.

No se encontraron diferencias en la puntuación en el IDB al ingreso entre los dos grupos. Sin embargo, el grupo que abandonó tuvo una puntuación significativamente menor en las escalas de trastornos ansiosos y somatoformes.

De igual manera, se encontraron diferencias significativas en la satisfacción con el servicio entre los dos grupos de mujeres. Las que abandonaron el programa señalaron menor satisfacción con el tratamiento prescrito y con el trato personal recibido. No hubo diferencias en cuanto a la satisfacción con los lugares donde se habían atendido.

A este respecto destacan los datos siguientes: a) la tasa de abandono a los tres meses fue de una de cada cinco mujeres $(20 \%)$ que ingresaron en el pro-

CUADRO 1. Comparación entre la puntuación en el inventario de depresión de Beck (IDB) y el diagnóstico registrado en la ficha clínica, en mujeres que ingresan en el programa de Detección, Diagnóstico y Tratamiento Integral de la Depresión $(n=141)$

\begin{tabular}{|c|c|c|c|c|c|c|}
\hline \multirow[b]{3}{*}{ Categorías del IDB } & \multicolumn{4}{|c|}{ Diagnóstico en ficha clínica } & & \\
\hline & \multicolumn{2}{|c|}{$\begin{array}{c}\text { Depresión } \\
\text { leve }\end{array}$} & \multicolumn{2}{|c|}{$\begin{array}{l}\text { Depresión } \\
\text { moderada }\end{array}$} & \multicolumn{2}{|c|}{ Total } \\
\hline & $n$ & $\%$ & $n$ & $\%$ & $n$ & $\%$ \\
\hline Sin depresión & 3 & 15,0 & 4 & 3,3 & 7 & 5,0 \\
\hline Depresión leve a moderada & 8 & 40,0 & 17 & 14,0 & 25 & 17,7 \\
\hline Depresión moderada a grave & 5 & 25,0 & 45 & 37,2 & 50 & 35,5 \\
\hline Depresión grave & 4 & 20,0 & 55 & 45,5 & 59 & 41,8 \\
\hline Total & 20 & 100,0 & 121 & 100,0 & 141 & 100,0 \\
\hline
\end{tabular}

CUADRO 2. Comparación de características sociales, clínicas y de satisfacción con la atención recibida, entre las mujeres que abandonan y permanecen en el programa de Detección, Diagnóstico y Tratamiento Integral de la Depresión

\begin{tabular}{|c|c|c|c|c|}
\hline \multicolumn{2}{|c|}{ Características } & \multicolumn{2}{|c|}{ Resultados } & \multirow[b]{2}{*}{ Valor $P$} \\
\hline Variable & Dimensiones & $\begin{array}{l}\text { Grupo que } \\
\text { abandona }\end{array}$ & $\begin{array}{l}\text { Grupo que } \\
\text { permanece }\end{array}$ & \\
\hline \multicolumn{5}{|l|}{ Sociales } \\
\hline Edad & $\begin{array}{l}\text { - promedio } \\
\text { - } \mathrm{DE}^{\mathrm{a}}\end{array}$ & $\begin{array}{l}38,8 \\
11,9\end{array}$ & $\begin{array}{l}45,4 \\
11,1\end{array}$ & 0,0027 \\
\hline Estado civil & $\begin{array}{l}\text { - con pareja } \\
\text { - sin pareja }\end{array}$ & $\begin{array}{l}21,1 \% \\
16,4 \%\end{array}$ & $\begin{array}{l}78,9 \% \\
83,6 \%\end{array}$ & 0,7764 \\
\hline Años de estudio & - mediana & 8 & 8 & 0,9641 \\
\hline Ocupación & $\begin{array}{l}\text { - con empleo } \\
\text { - sin empleo }\end{array}$ & $\begin{array}{l}15,4 \% \\
21,4 \%\end{array}$ & $\begin{array}{l}84,6 \% \\
78,6 \%\end{array}$ & 0,3652 \\
\hline $\begin{array}{l}\mathrm{N}^{\circ} \text { de condiciones } \\
\text { psicosociales de riesgo }\end{array}$ & - mediana & 2 & 3 & 0,0159 \\
\hline \multicolumn{5}{|l|}{ Clínicas } \\
\hline $\mathrm{IDB}^{\mathrm{b}}$ & $\begin{array}{l}\text { - promedio } \\
\text { - DE }\end{array}$ & $\begin{array}{l}25,4 \\
10,3\end{array}$ & $\begin{array}{r}26,2 \\
9,9\end{array}$ & 0,6943 \\
\hline Trastornos de ansiedad & $\begin{array}{l}\text { - promedio } \\
\text { - DE }\end{array}$ & $\begin{array}{r}12,6 \\
5,0\end{array}$ & $\begin{array}{r}14,9 \\
5,0\end{array}$ & 0,0155 \\
\hline $\begin{array}{l}\text { Trastornos } \\
\text { somatoformes }\end{array}$ & $\begin{array}{l}\text { - promedio } \\
\text { - DE }\end{array}$ & $\begin{array}{l}9,0 \\
4,3\end{array}$ & $\begin{array}{r}10,9 \\
4,9\end{array}$ & 0,0385 \\
\hline \multicolumn{5}{|l|}{ Satisfacción con la atención } \\
\hline $\begin{array}{l}\text { Con el tratamiento } \\
\text { prescrito }\end{array}$ & $\begin{array}{l}\text { - promedio } \\
\text { - } \mathrm{DE}\end{array}$ & $\begin{array}{l}6,5 \\
2,8\end{array}$ & $\begin{array}{l}8,7 \\
2,1\end{array}$ & 0,0000 \\
\hline $\begin{array}{l}\text { Con el lugar } \\
\text { de atención }\end{array}$ & $\begin{array}{l}\text { - promedio } \\
\text { - DE }\end{array}$ & $\begin{array}{l}8,2 \\
2,3\end{array}$ & $\begin{array}{l}8,8 \\
1,7\end{array}$ & 0,3372 \\
\hline $\begin{array}{l}\text { Con el trato } \\
\text { recibido }\end{array}$ & $\begin{array}{l}\text { - promedio } \\
\text { - DE }\end{array}$ & $\begin{array}{l}8,1 \\
2,1\end{array}$ & $\begin{array}{l}9,3 \\
1,5\end{array}$ & 0,0000 \\
\hline
\end{tabular}

${ }^{a} D E$, desviación estándar.

b IDB, inventario de depresión de Beck.

grama, b) estas mujeres atribuían su deserción principalmente a razones personales y al tipo de tratamiento, y c) las mujeres que abandonaron el programa tendían a ser más jóvenes, a pre- sentar menos factores psicosociales de riesgo, menos comorbilidad ansiosa y somatomorfa y a manifestar mayor insatisfacción con la atención clínica y personal que recibieron. 


\section{Adherencia al tratamiento y cambios en el cuadro clínico a los tres meses}

En el grupo de 136 pacientes (80,5\%) que prosiguieron en el PDTID se analizaron el tipo de tratamiento prescrito y la adherencia a la prescripción. De ese total, a 131 mujeres $(96,3 \%)$ se les prescribió tratamiento con fármacos antidepresivos. De las cinco restantes $(3,7 \%)$, a tres se les indicó intervención grupal y a dos una combinación de esta y de psicoterapia individual. En este subgrupo sin tratamiento farmacológico, la puntuación en el IDB fue significativamente menor que en el que recibió prescripción de antidepresivos (promedio en el IDB: 14,6 puntos frente a 26,6 puntos, respectivamente) $(P=0,0072)$.

En el cuadro 3 se muestra la distribución de los tipos de tratamiento que se indicaron a las 131 pacientes tratadas con psicofármacos: en $21,4 \%$ solo se prescribieron antidepresivos, en 7,6\% se prescribió además psicoterapia individual, en $36,6 \%$ se pidió integrarse a terapia de grupo y en $34,4 \%$ se prescribió una combinación de psicoterapia individual y grupal. No se encontraron diferencias significativas en la puntuación del IDB entre estos cuatro grupos ( $P=0,3182)$, lo que demuestra que el criterio aplicado para prescribir alguna de las opciones de tratamiento no fue la gravedad del cuadro depresivo.

$\mathrm{Al}$ estudiar la adherencia a los distintos tipos de tratamiento se obtuvieron los datos siguientes, a los tres meses de seguimiento:

- De las 131 mujeres que recibieron prescripción de antidepresivos, 73,3\% habían cumplido correctamente las indicaciones y las demás solo las siguieron una parte del tiempo o en forma intermitente $(26,7 \%)$.

- De las 98 mujeres a quienes se prescribió la intervención grupal, 17,3\% nunca acudieron, 37,8\% lo hicieron a todas las sesiones y $44,9 \%$ tuvieron participación irregular.

- De las 57 mujeres que se remitieron a psicoterapia individual, 12,3\% nunca asistieron, 47,4\% acudieron a todas las sesiones y 40,4\% lo hicieron en forma irregular.

CUADRO 3. Distribución de los tipos de tratamiento que se prescribieron al grupo de 131 pacientes que recibían antidepresivos y sus respectivas puntuaciones en el Inventario de Depresión de Beck

\begin{tabular}{lrrrr}
\hline \multirow{2}{*}{\multicolumn{1}{c}{ Tipos de tratamiento }} & \multicolumn{2}{c}{ Casos } & \\
\cline { 2 - 3 } \multicolumn{1}{c}{$n$} & $n$ & $\%$ & Puntuación del IDB \\
\hline Solo antidepresivos & 28 & 21,4 & $26,0 \pm 9,0$ \\
Con psicoterapia individual & 10 & 7,6 & $23,2 \pm 10,0$ \\
Con intervención grupal & 48 & 36,6 & $28,5 \pm 9,1$ \\
Psicoterapia individual e intervención grupal & 45 & 34,4 & $25,7 \pm 10,5$ \\
Total & 131 & 100,0 & $26,6 \pm 9,7$ \\
\hline
\end{tabular}

Si la adherencia se mide como el cumplimiento cabal de las indicaciones por cada tratamiento, cabe concluir que corresponde a los antidepresivos la adherencia más alta (casi 75\% de las pacientes), seguidos de la psicoterapia individual (casi 50\%) y, por último, de la intervención grupal (poco más de un tercio de las pacientes).

En cuanto a los cambios clínicos observados en el cuadro depresivo entre la entrevista inicial y la de seguimiento a los tres meses, la puntuación en el IDB disminuyó en $77,2 \%$ de las pacientes y se mantuvo estable en $3,7 \%$. Si se considera el grupo completo, la puntuación promedio en el IDB descendió desde 26,2 $\pm 9,9$ puntos en la valoración inicial hasta $18,5 \pm 10,3$ puntos al final del seguimiento, lo que hace una diferencia promedio de $7,7 \pm 10,8$ puntos, la cual resulta estadísticamente significativa $(P<0,0001)$.

En el cuadro 4 pueden apreciarse las variaciones de acuerdo con la gravedad del cuadro clínico (según la puntuación del IDB al ingreso). Es evidente que las pacientes con trastornos más graves presentaron mayor descenso en la puntuación del IDB. La va- riación fue significativamente mayor en el grupo de "depresión grave" que en todos los demás $(P<0,0001)$. En un análisis de regresión lineal entre la puntuación inicial del IDB y la diferencia entre las puntuaciones de las dos entrevistas, se encontró un valor $r=$ 0,51 , con $r^{2}=0,26$ (IC95\% entre 0,09 y $0,41)$, lo que viene a confirmar que los mejores resultados se obtuvieron en las mujeres con cuadros más graves.

La evaluación de la comorbilidad indica que se produjo un descenso significativo también en las puntuaciones promedio de los síntomas ansiosos (de 14,9 $\pm 5,0$ puntos a $11,7 \pm 6,0$ puntos, con un valor $P<0,0001)$ y en los síntomas somatomorfos (de 10,9 \pm 4,9 puntos a $8,8 \pm 5,2$ puntos, con un valor $P<0,0001$ ).

Las conclusiones de este apartado serían que: a) los equipos utilizan todos los tipos de tratamientos disponibles (fármacos, psicoterapia e intervención grupal), con primacía de los psicofármacos; b) la adherencia es buena al uso de antidepresivos y moderada al de la psicoterapia y la intervención grupal; c) el grupo tratado presenta una reducción significativa

\begin{abstract}
CUADRO 4. Variación en la puntuación del Inventario de Depresión de Beck entre la entrevista inicial y a los tres meses de seguimiento, por grupos, según la gravedad del cuadro depresivo en la valoración inicial
\end{abstract}

\begin{tabular}{lcr}
\hline \multicolumn{1}{c}{$\begin{array}{c}\text { Cuadro depresivo } \\
\text { (según puntuación en el IDB al ingreso) }\end{array}$} & $\begin{array}{c}\text { Promedio de } \\
\text { variación en el IDB }\end{array}$ & $\begin{array}{c}\text { Desviación } \\
\text { estándar }\end{array}$ \\
\hline Depresión leve a moderada & 3,48 & 6,04 \\
Depresión moderada a grave & 5,44 & 8,09 \\
Depresión grave & 13,15 & 12,57 \\
\hline
\end{tabular}


de la intensidad del cuadro depresivo después de tres meses, efecto que es más notorio en quienes ingresan con cuadros más graves, y d) a los tres meses de tratamiento se aprecia también una reducción significativa de los síntomas ansiosos y somatoformes.

\section{DISCUSIÓN}

De acuerdo con el planteamiento de W. Katon et al. (19), la estrategia de desarrollar un programa de carácter comunitario para la detección y tratamiento de la depresión es buen mecanismo para reducir la prevalencia de esta enfermedad y el impacto que genera en las personas, la familia y la sociedad en su conjunto. Sin embargo, la eficacia que muestran los tratamientos en ensayos clínicos controlados no siempre se traduce en una efectividad semejante de los programas aplicados, ya sea por factores que afectan a la validez externa de dichos estudios o por aspectos inherentes a las personas atendidas, a las intervenciones, a los prestadores de los servicios o a las formas en que se organiza la atención.

Se han identificado problemas en la detección de los casos de trastorno depresivo por parte del equipo de atención primaria. Asimismo, no siempre es adecuada la prescripción que hacen de medicamentos o de intervenciones psicosociales, al tiempo que solo un porcentaje de los pacientes cumple con estos tratamientos por el tiempo establecido $(6,20)$. Estas razones han motivado la instauración de programas con capacitación de los profesionales, provisión de recursos específicos para atender estos problemas y cambios en la organización del servicio, según se ha llevado adelante en Chile (14). Esta evaluación preliminar permite conocer cómo está funcionando el programa y qué resultados logra en las personas atendidas.

En primer lugar, se demostró que la gran mayoría de las pacientes que ingresaron en el programa $(95,2 \%)$ padecía un cuadro depresivo, lo cual indica que la identificación del caso se produce de forma correcta y que esta vía no se utiliza para atender problemas psicosociales que no corresponden a este cuadro clínico. Sin embargo, la precisión para valorar la intensidad de los síntomas depresivos fue cercana a $50 \%$, con tendencia a subestimar la magnitud del trastorno. Este resultado puede reflejar una capacitación subóptima para la evaluación clínica de estos casos, o bien ser una expresión administrativa de la dificultad para derivar los casos más complejos a la atención especializada; en otras palabras, se diagnostica un cuadro menos grave para eludir la normativa de derivación correspondiente a fin de poder ofrecer atención, aun cuando no sea especializada. Otra explicación probable sería la discordancia inherente al uso de un instrumento de tipo dimensional (el IDB) para evaluar una estrategia diagnóstica de tipo categórico (el diagnóstico clínico). Con tal de obviar esa limitación, en estudios futuros debería incorporarse una entrevista clínica estandarizada que sea aplicable por el evaluador externo.

La tasa de abandono del programa, de aproximadamente $20 \%$, es menor que la que informan otros estudios; en dos de ellos, por ejemplo, se indica que cerca de un tercio de los pacientes dejan de tomar los antidepresivos antes de completar el mes de tratamiento $(6,21)$. En el caso presente debe considerarse que cierto porcentaje pudo no llegar a la entrevista de evaluación inicial y abandonar el tratamiento después de la primera indicación, con lo que la tasa de abandono podría ser mayor que la estimada. También es posible que parte de la muestra inicial a la que no se pudo contactar para la segunda entrevista haya abandonado las visitas de seguimiento, lo que significaría un aumento sobre la tasa de abandono calculada. Este aspecto deberá someterse a evaluaciones posteriores.

En todo caso, sería posible reducir la cifra de abandono si se mejorara la flexibilidad con que los equipos manejan las indicaciones y organizan sus servicios. Es una medida importante, porque al elaborar normativas nacionales se corre el riesgo de afectar a la necesaria adaptación que deben hacer los equipos locales a cada realidad, un es- fuerzo en que consideran sus propias condiciones, capacidades y recursos, además de aspectos socioculturales de su comunidad. La imprevisión en este sentido conlleva el riesgo de reducir la flexibilidad de la atención, la creatividad de los equipos para promover la adherencia al tratamiento, la adecuación a cada caso en particular y la satisfacción que el paciente obtiene de los servicios.

Sin embargo, las pacientes que abandonaron este programa presentaban menor complejidad clínica y psicosocial (menos comorbilidad, menor edad y menos situaciones psicosociales de riesgo). El seguimiento que logró hacerse de estas pacientes a los tres meses mostró una reducción significativa de los síntomas depresivos, a pesar de no estar en tratamiento. Esta situación podría deberse a una autoselección de los casos, que trae consigo un mejor pronóstico respecto a la evolución de la enfermedad. Estudios ulteriores deberían realizar seguimientos más prolongados para definir qué sucede con los síntomas y la tasa de recaídas o de evolución crónica que pudieran presentarse, lo cual redundaría negativamente en la carga que esta enfermedad representa para la comunidad. Hay pruebas suficientes en el sentido de que el riesgo de una peor evolución subsiguiente aumenta cuando no se trata adecuadamente el episodio depresivo $(22,23)$, así como de un mayor grado de discapacidad (24) y de utilización de servicios médicos (25) en relación con la persistencia de síntomas depresivos.

El análisis de los tipos de tratamiento prescritos indica que la elección entre fármacos e intervenciones psicosociales (individuales o grupales) no guardó relación con la intensidad del trastorno depresivo, con excepción del pequeño grupo al que no se le prescribieron antidepresivos, el cual, por cierto, presentó una puntuación significativamente más baja en el IDB. Esta línea de acción difiere de lo que propone la normativa del programa, lo que obliga a evaluar con mayor precisión este punto en estudios futuros, a fin de verificar la efectividad y eficiencia con que se están utilizando los re- 
cursos, considerando cada intervención en particular.

La adherencia completa a los tratamientos fue de $73,3 \%$ en el caso de los fármacos, de $47,4 \%$ en el de la psicoterapia individual y de $37,8 \%$ en el de la intervención grupal. Estos datos ponen de relieve la necesidad de desarrollar estrategias específicas destinadas a mejorar la adherencia a los tratamientos, en particular a los de tipo psicosocial, que tienen una inasistencia cercana a $15 \%$ y una asistencia irregular de $40 \%$.

Los resultados acerca del cuadro depresivo indican que las pacientes que se atienden en el programa presentan una mejoría importante: en $77,2 \%$ disminuyó la puntuación en el IDB; en el grupo completo se observó un descenso promedio de 7,7 puntos (cerca de un tercio de la puntuación basal), un cambio positivo que fue más notable en el grupo que presentaba depresión más grave. Estos resultados concuerdan con los publicados acerca de grupos de pacientes sometidos a tratamientos tradicionales $(6,8)$. En el futuro será necesario mejorar los resultados mediante la instauración de medidas específicas para incrementar la adherencia a los tratamientos e intervenciones (11). Sería importante también identificar al grupo con peor pronóstico, con el fin de ofrecerle un programa más intensivo o manejar los casos en forma más individualizada.

Otro dato relevante fue el descenso de los síntomas somatomorfos y ansiosos (ante todo estos últimos) en proporción directa con los síntomas depresivos. Esta observación refuerza la hipótesis de que los síntomas de este tipo guardan estrecha relación entre sí, de modo que tienden a conformar un solo cuadro clínico y no dos o más que están presentes simultáneamente (comorbilidad) (26-28). Cabe también postular que haya un "efecto de amplio espectro" de los tratamientos del programa, en el que los mecanismos psicobiológicos subya- centes serían los mismos o estarían estrechamente interconectados.

En definitiva, la evaluación del período de puesta en marcha de este programa instaurado por el Ministerio de Salud de Chile muestra que se están cumpliendo sus objetivos generales y que constituye una estrategia adecuada para enfrentar el problema referente a la detección, diagnóstico y tratamiento de la depresión. En la etapa siguiente será necesario ahondar en temas más específicos, como la precisión del diagnóstico clínico o la adherencia a los tratamientos, con estrategias específicas para mejorar estos aspectos. En futuras evaluaciones deberá considerarse un seguimiento más prolongado de los casos (en tratamiento y en abandono) y un análisis de la efectividad (general y de intervenciones específicas), así como de la organización y funcionamiento de los equipos (por ejemplo, la evaluación de "buenas prácticas").

\section{REFERENCIAS}

1. Geddes J, Butler R, Hatcher S. Depressive disorders. En: BMJ Publishing Group: clinical evidence mental health. Londres: BMJ Publishing Group Limited; 2002;84-106.

2. Stevens L, Kinmonth AL, Peveler RC, Thompson C. The Hampshire Depression Project: development and piloting of clinical practice guidelines and education about depression in primary health care. Med Educ. 1997; 31:375-9.

3. UK Department of Health. Treatment choice in psychological therapies and counseling. Evidence based clinical practice guideline. Londres: UK Department of Health Publications; 2001.

4. Campbell D, Stanley J. Diseños experimentales y cuasiexperimentales en la investigación social. Buenos Aires: Ed. Amorrortu; 1973.

5. Wells K. Treatment research at the crossroads: the scientific interface of clinical trials and effectiveness research. Am J Psychiatry. 1999; 156(1):5-10.

6. Katon W, Von Korff M, Lin E, Walter E, Simon GE, Bush T, et al. Collaborative management to achieve treatment guidelines: impact on depression in Primary Care. JAMA. 1995; 273(13):1026-31.

7. Rutz W, Walinder J, Eberhard G, Holmberg G, von Knorring AL, von Knorring L, et al. An educational program on depressive disorders for general practitioners on Gotland: background and evaluation. Acta Psychiatr Scand. 1989;79:19-26.
8. Sherbourne CD, Wells KB, Duan N, Miranda $\mathrm{J}$, Unützer J, Jaycox L, et al. Long-term effectiveness of disseminating quality improvement for depression in primary care. Arch Gen Psychiatry. 2001;58:696-703.

9. Thompson C, Kinmonth AL, Stevens L, Peveler RC, Stevens A, Ostler KJ, et al. Effects of a clinical-practice guideline and practicebased education on detection and outcome of depression in Primary Care: Hampshire Depression Project randomised controlled trial. Lancet. 2000;355:185-91.

10. Katon W, Rutter C, Ludman EJ, Von Korff M Lin E, Simon GE, et al. A randomised trial of relapse prevention of depression in Primary Care. Arch Gen Psychiatry. 2001;58:241-7.

11. Gilbody S, Whitty P, Grimshaw J, Thomas R. Educational and organizacional interventions to improve the management of depression in Primary Care. JAMA. 2003;289(23): 3145-51.

12. Badamgarav E, Weingarten SR, Henning JM, Knight K, Hasselblad V, Gano A, et al. Effectiveness of disease management programs in depression: a systematic review. Am J Psychiatry. 2003;160(12):2080-90.

13. Araya R, Rojas G, Fritsch R, Gaete J, Rojas M, Simon $\mathrm{G}$, et al. Treating depression in primary care in low-income women in Santiago, Chile: a randomised controlled trial. Lancet. 2003; 361:995-1000

14. Chile, Ministerio de Salud. Guía clínica para la atención primaria. La depresión: detección, diagnóstico y tratamiento. Santiago, Chile: Unidad de Salud Mental y Departamento de Modelo de Atención del Ministerio de Salud; 2001.

15. Beck AT, Ward CH, Mendelson M, Mock J, Erbaugh J. An inventory for measuring depression. Arch Gen Psychiatry. 1961;4:53-63.

16. Beck AT, Steer RA. Beck Depression Inventory (BDI). En: American Psychiatric Association. Handbook of psychiatric measures. Washington, D.C.: APA; 2000;519-23.

17. Beck AT, Steer RA, Gabin MG. Psychometric properties of the Beck Depression Inventory: twenty-five years of evaluation. Clin Psychol Rev. 1988:8:78-100.

18. Alvarado R, Vera A, Toledo MI, Serrano F, Gatica CG, Núñez C, et al. Validación de escalas para medir trastornos depresivos, por ansiedad y somatoformes. Cuadernos de Psicología № 2. Santiago, Chile: Universidad Diego Portales; 1992.

19. Katon $W$, Von Korff $M$, Lin E, Unützer J, Simon G, Walker E, et al. Population-based care of depression: effective disease management strategies to decrease prevalence. Gen Hosp Psychiatry. 1997;19:169-78.

20. Katon W. Will improving detection of depression in primary care lead to improved depressive outcomes? Gen Hosp Psychiatry. 1995;17:1-2.

21. Katon W, Robinson P, Von Korff M, Lin E, Bush T, Ludman E, et al. A multifaceted intervention to improve treatment of depression in 
primary care. Arch Gen Psychiatry. 1996;53: 924-30.

22. Melfi CA, Charla AJ, Croghan Th, Hanna MP, Kennedy S, Sredl K. The effects of adherence to antidepressant treatment guidelines on relapse and recurrence of depression. Arch Gen Psychiatry. 1998;55:1128-32.

23. Delgado PL. Approaches to the enhancement of patient adherence to antidepressant medication treatment. J Clin Psychiatry. 2000;61 (suppl 2):6-9.

24. Wells KB, Stewart A, Hays RD, Burnam A, Rogers W, Daniels $M$, et al. The functioning and well-being of depressed patients: results from the Medical Outcomes Study. JAMA. 1989;262:914-9.

25. Johnson I, Weissman MM, Klerman GL. Service utilization and social morbidity associated with depressive symptoms in the community. JAMA. 1992;267:1478-83.

26. Andrews G, Stewart G, Morris-Yates A, Holt $\mathrm{Ph}$, Henderson S. Evidence for a general neurotic syndrome. Br J Psychiatry. 1990;157: 6-12.

27. Sullivan PF, Kendler KS. Typology of common psychiatric syndromes. An empirical study. Br J Psychiatry. 1998;173:312-9.
28. Ojeda C. Angustia y depresión: ¿comorbilidad o cuadros clínicos complejos? Rev Psiquiatría. (Sociedad Chilena de Salud Mental) 2003;19 (2):120-6.

Manuscrito recibido el 28 de septiembre de 2004. Aceptado para publicación, tras revisión, el 19 de agosto de 2005.

ABSTRACT Objective. To conduct a preliminary evaluation of the Program for Depression Detection, Diagnosis, and Comprehensive Treatment, which was developed by the Ministry of Health of Chile.

\section{Evaluation of the Program for Depression Detection, Diagnosis, and Comprehensive Treatment in primary care in Chile}

Material and methods. A group of women who had entered the Depression Program in eight primary care centers in three regions of Chile were monitored for three months. The initial sample was made up of 229 women, of whom 169 were able to be interviewed at the end of the three months. Sociodemographic variables, psychosocial risk factors, anxiety and somatic symptoms, and the degree of satisfaction with the care received were studied. Depressive symptoms were evaluated with the Beck Depression Inventory (BDI). The treatments prescribed for the women were also analyzed.

Results. The clinicians' decision to enroll the patients in the Depression Program was appropriate, with $95.2 \%$ of the women having depressive symptoms, according to the BDI. The accuracy in the clinical assessment of the severity of the symptoms was around $50 \%$, with a tendency to underestimate the severity of the depression.

The dropout rate for the women was $19.5 \%$ at the end of three months of monitoring. Those who dropped out tended to present less severe clinical symptoms (less intense anxiety and somatic symptoms), a smaller number of psychosocial risk factors, and a lower level of satisfaction with the care received.

The patients showed good adherence (73.3\%) with the pharmacological treatment, but less with the individual psychotherapy $(47.4 \%)$ and the group psychotherapy $(37.8 \%)$. A significant decline was seen in the intensity of the depressive symptoms by the end of the three months, with the decline being greater among the women with more serious symptoms. There was also a significant decline in anxiety and somatic symptoms.

Conclusions. The preliminary evaluation indicates that the Depression Program is meeting the proposed objectives, although there appears to be a need to develop specific strategies to improve the accuracy of the clinical diagnosis as well as compliance with the treatments that are offered.

Key words Program evaluation, depressive disorder, Chile.
El viaje más largo es el que se hace hacia el interior de uno mismo.

Day Hammarskjöld,

ex Secretario de las Naciones Unidas (1905-1961) 